\title{
Trans-Ulnar Vein Graft PCI in an Octogenarian
}

\author{
Asrar Ahmed ${ }^{1}$, Nora Rossberg ${ }^{2}$ and ${ }^{*}$ Ahmed Elghamaz ${ }^{3}$ \\ Department of Cardiovascular Medicine, UK
}

Submission: November 23, 2016; Published: December 07, 2016

*Corresponding author: Ahmed Elghamaz MRCP; Consultant (Interventional) Cardiologist; Department of Cardiovascular Medicine; Northwick Park Hospital, Harrow; United Kingdom, HA1 3UJ, UK

\begin{abstract}
Abbreviations: LIMA : Left Internal Mammary Artery; LAD: Left Anterior Descending artery; ACS: Acute Coronary Syndrome; SVG: Saphenous
\end{abstract} Vein Graft; PCI: Percutaneous Intervene; DES: Drug Eluting Stents

\section{Case Report}

An 86-year-old gentleman was admitted to our cardiac centre with chest pain and a troponin I rise to $4.85 \mathrm{ng} / \mathrm{ml}$ (normal value $<0.03 \mathrm{ng} / \mathrm{ml}$ ). His serial ECGs were unremarkable for any dynamic changes. Significant past history included coronary artery bypass graft in 1985 with presumed four grafts of unknown anatomy. Two years back, he was admitted with a collapse secondary to complete heart block requiring a permanent pacemaker. Coronary angiogram prior to pacemaker insertion was challenging with failed femoral access on both sides. Finally, angiography via left radial route revealed severe native vessel disease and a patent vein graft to the left circumflex. The operators were unable to incubate and visualize the left internal mammary artery (LIMA) to left anterior descending artery (LAD) graft and the remaining vein grafts despite multiple attempts with different catheters.

The diagnostic procedure at that time involved high contrast load (300 $\mathrm{ml}$ of Visipaque) and hence the procedure was stopped and the decision made to manage the patient medically. The patient's representation to us this time was managed as non-ST elevation acute coronary syndrome (ACS). An echocardiogram revealed a normal left ventricular ejection fraction (55-60\%) and severe hypokinesia of the antero-septal segments. After discussion with the patient, decision was taken to repeat coronary angiography albeit keeping in mind pitfalls from the previous procedure. The access site again posed a challenge for us, as there were no femoral pulses palpable. Being a graft study, the right radial artery was not attractive while the left radial artery was barely palpable (used for access previously).

The only suitable remaining access site was the left ulnar artery. Using the standard preparation technique for trans-radial access, the left ulnar artery was punctured just proximal to the pisiform bone with a 22-gauge needle and Merit $6 \mathrm{~F}$ hydrophilic sheath was inserted using standard Seldinger's technique. Heparin $5000 \mathrm{U}$ and nitroglycerin $200 \mathrm{mcg}$ was given as a cocktail through the sheath. The left internal mammary artery (LIMA) was cannulated easily with 5F JR4 diagnostic catheter and was not a graft vessel (Figure 1). The right coronary artery was non-dominant and chronically occluded. 5F AL-1 diagnostic catheter was used to visualize the left coronary system and the vein grafts. The left coronary artery had severe native disease. The saphenous vein graft (SVG) to left circumflex (obtuse marginal) was patent with good distal run-off while another saphenous vein graft to LAD (which was not seen before) had critical proximal sub-total occlusion (likely culprit vessel) (Figures 2 \& 3).

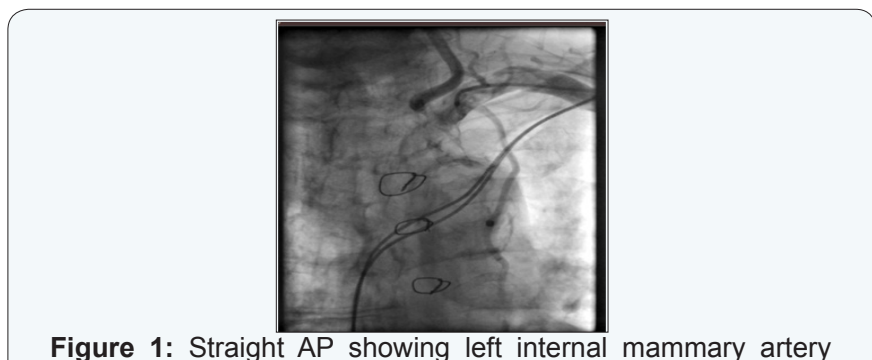
(which was not a graft vessel).

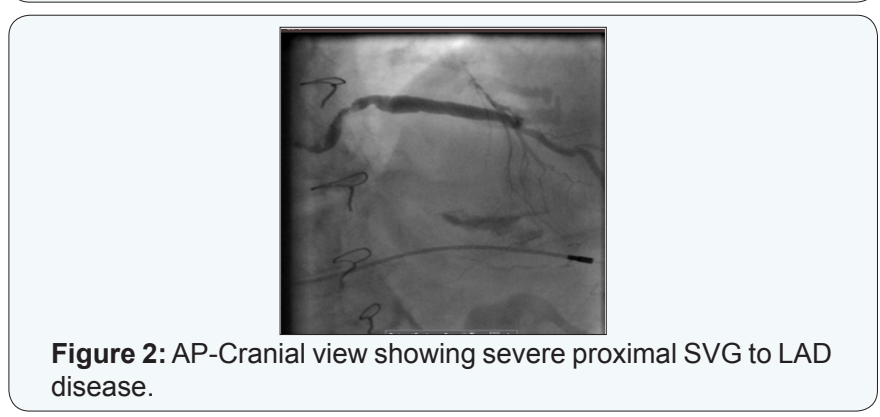




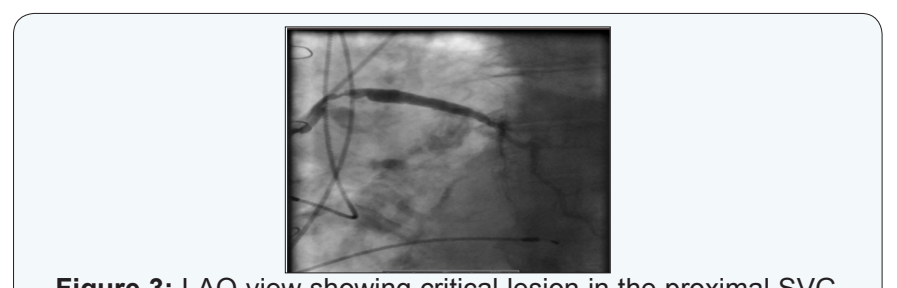

Figure 3: LAO view showing critical lesion in the proximal SVG to LAD.

We decided to percutaneously intervene (PCI) on the proximal SVG-to-LAD lesion. Using 6F AL1 guide, sion blue wire crossed the lesion easily and the lesion was pre-dilated with $3 \times 15 \mathrm{~mm}$ non-compliant balloon keeping in view the calcific nature of the stenosis. Two Ultimate drug eluting stents (DES) $4 \times 33 \mathrm{~mm}$ and $4 \times 15 \mathrm{~mm}$ were deployed to cover the lesion up to the ostium of the vein graft. Both were post-dilated with $4.5 \times 12$ and then $5 \times 12 \mathrm{~mm} \mathrm{NC}$ at 20 atmospheres. The final angiogram showed some residual under-expansion due to heavily calcific disease but otherwise excellent result (Figures 4-6). Transradial band was applied over the left wrist. The ulnar artery was patent by palpation at 1- and 7-day follow up.
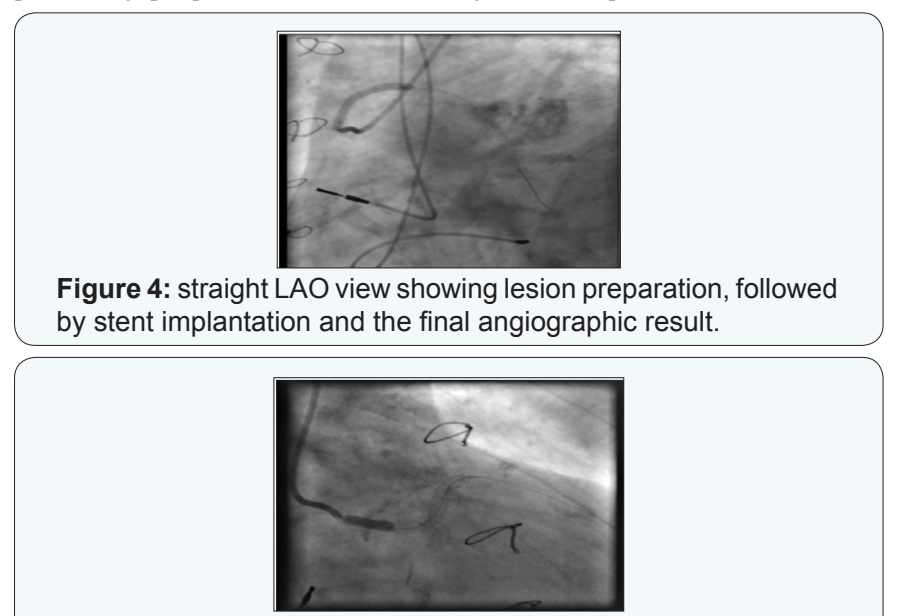

Figure 5: straight RAO view showing lesion preparation, followed by stent implantation and the final angiographic result.

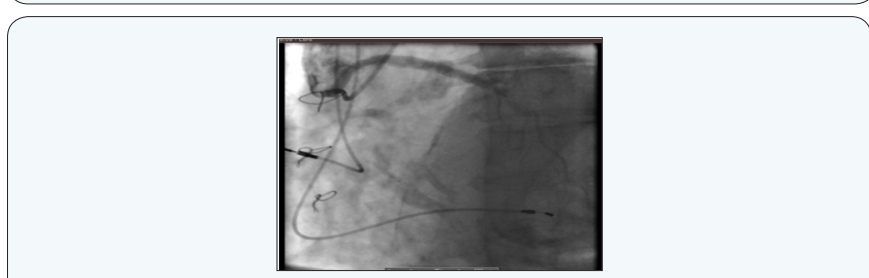

Figure 6: LAO view showing lesion preparation, followed by stent implantation and the final angiographic result.

\section{Discussion}

To the best of our knowledge, this is a unique case illustrating left trans-ulnar vein graft PCI in an octogenarian in the context of an ACS. The majority of trans-ulnar cardiac catheterizations are diagnostic in nature and data is limited to only around 2,500 $[1,2]$ since Terashima et al. [3] first published their experience on nine patients more than a decade ago [3]. Only a limited number of PCIs are being performed via the ulnar artery, mostly for failed trans-radial access.

This includes failed radial artery puncture, radial artery loops and curvatures, radial spasm or hypoplastic radial artery. Other indication for performing a trans-ulnar cardiac catheterization includes patients undergoing bypass surgery with use of radial artery grafts. By far, it is still not a default route even for advanced radial operators. Current ACC/AHA/ESC/SCAI guidelines (2011) do not even mention the potential use of ulnar access for cardiac catheterization and coronary interventions.

\section{Learning Point}

* The ulnar artery can be used as an alternative access for vein graft PCI in patients not suitable for trans-femoral and trans-radial approaches. Age and clinical presentation should not hinder the procedure in appropriately selected patients. It would be appropriate to establish a registry on all transulnar PCIs so that all procedures are appropriately audited and outcomes measured.

\section{References}

1. Sattur S, Singh M, Kaluski E (2014) Transulnar access for Coronary angiography and Percutaneous Coronary Interventions. J Invasive Cardiol 26(8): 404-408.

2. Gokhroo R, Bisht D, Padmanabhan D, Gupta S, Kishor K, al (2015) Feasibility of ulnar artery catheterization: Ajmer Ulnar Artery (AJULAR) catheterization study. Catheter Cardiovasc Interv 86(1): 4248.

3. Terashima M, Meguro T, Takeda H, Endoh N, Ito Y, et al. (2001) percutaneous ulnar artery approach for coronary angiography: a preliminary report in nine patients. Catheter Cardiovasc Interv 53(3):

410-414.

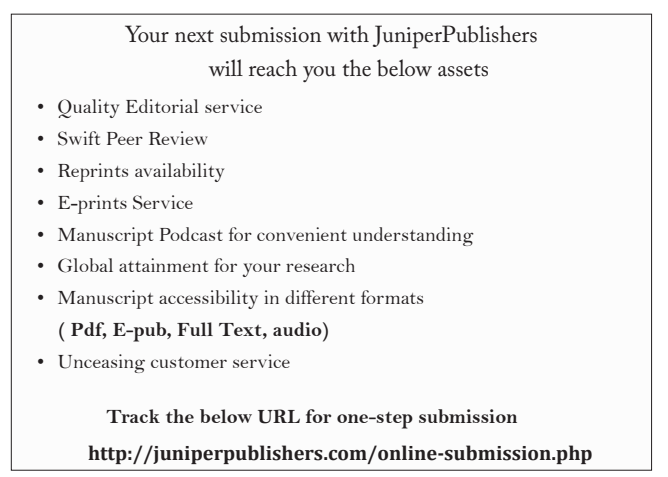

